\title{
Performance Measures for Image Fusion based on Top-hat Transform and Wavelet Transform
}

\author{
Chen Songchao $^{1}$, Dr. B. Sujatha ${ }^{2}$ and G. Karuna ${ }^{3}$ \\ ${ }^{1}$ Department of Information and Communication, Guilin University of Electronic \\ Technology, Guilin, Guangxi, China \\ ${ }^{2}$ Professor, Dept. of CSE, GIET, Rajahmundry \\ ${ }^{3}$ Research Scholar, GRIET, Hyderabad \\ Ichemmysc0124@126.com, ${ }^{2}$ birudusujatha@gmail.com, ${ }^{3}$ karunavenkatg@gmail.com
}

\begin{abstract}
Image fusion is a process in which a high-resolution Panchromatic Image (PAN) is combined with a low-resolution Multispectral Image (MS) to form a new single image that contains both the spatial information of the PAN image and the spectral information of the $M S$ image. In the present work, an algorithm for image fusion based on the Wavelet Transform (WT) is implemented, analyzed, and compared with the top-hat transform algorithm. The decimated and undecimated wavelets used in image fusion can be categorized into three classes: Orthogonal, Biorthogonal, and Nonorthogonal. Fusion results are evaluated and compared using various measures of performance and the results show that the undecimated biorthogonal wavelet-based fusion method performs the fusion of PAN image and MS image better than top-hat transform fusion method, decimated orthogonally, decimated biorthogonal, and undecimated orthogonal wavelet-based fusion methods, especially in preserving both spectral and spatial information. The experiment is conducted on the IRS-1D images using LISS III scanner for the locations Vishakhapatnam and Hyderabad, India, and on Quick Bird image data and Losangels image data. The results show that the proposed WT fusion method works well in multi-resolution fusion and also preserves the original color or spectral characteristics of the input image data. In addition, the fused image has a better eye perception than the input ones.
\end{abstract}

Keywords: Image fusion, Top-hat transform, Wavelet transform, Decimated wavelets, Undecimated wavelets, Performance measures

\section{Introduction}

Image fusion is a tool where gray-level high-resolution PAN image is integrated with a colored low-resolution MS image to produce a fused image that retains the most desirable characteristics of PAN and MS images. The new image contains both the high-resolution spatial information of the PAN image and the spectral information of the MS image. The objective is to keep maximum spectral information from the original MS image while increasing the spatial resolution.

The fused image can have complementary spatial and spectral resolution characteristics. Image fusion methods can be broadly classified into two - spatial domain fusion and

Article history:

Received (January 10, 2014), Review Result (March 21, 2014), Accepted (May 5, 2014) 
transform domain fusion. The fusion methods which fall under spatial domain approaches such as averaging, Brovey method, Principal Component Analysis (PCA) method, Intensity Hue Saturation (IHS) method, etc., fall under spatial domain approaches. The disadvantage of spatial domain approaches is that they produce spatial distortion in the fused image. By using transform domain fusion techniques, spatial distortion can be very well handled by transform domain approaches in image fusion. Multiresolution analysis has become a very useful tool for analyzing remote sensing images. The various methods of image fusion using multiresolution techniques have been suggested before [1]-[4]. The fusion methods such as top-hat transform, Laplacian pyramid based, curvelet transform-based, discrete wavelet transform, etc., has become a very useful tool for image fusion. These methods show a better performance in the spatial and spectral quality of the fused image compared to other spatial methods of fusion.

The present paper concentrates on the fusion of satellite images. The panchromatic image is acquired by satellites with the maximum resolution with broad visual wavelength range but rendered in black and white. The multispectral data are transmitted with coarser resolution and acquired in more than one spectral or wavelength interval. This will usually be two or four times lower. At the receiver station, the panchromatic image is merged with the multispectral image data to convey more information.

In recent years, many papers about Image Fusion based on Wavelet Transform have been published. King and Wang (2001) introduced a wavelet-based sharpening method that uses IHS transformation and biorthogonal wavelet decomposition. Hong and Zhang (2003) integrated IHS and wavelets to fuse Quickbird images and IKONOS images and obtained promising results. Oguz Gungor and Jie Shan presented the Evaluation of Satellite Image Fusion using the Wavelet Transform method by testing Quickbird and IKONOS images. Richard B.Gomez, Amin Jazaeri, and Menas Kafatos proposed a Wavelet-based hyperspectral and multispectral image fusion between two spectral levels of a hyperspectral image and one band of multispectral image. Din-Chang Tseng, Yi-Ling Chen, and Michael S.C. Liu proposed integer Wavelet Transform and Principal Component Analysis (PCA) to fuse lowresolution Land sat TM Multispectral images and SPOT Panchromatic (PAN) Image to generate spectrum-preserving high-resolution Multispectral images. Qu Jishuang, Wang Chao used A Wavelet Package-based Data Fusion method for Multitemporal Remote Sensing Image Processing. G.Hong and Y.Zhang (2008) presented Comparision and Improvement of Wavelet-based Image fusion to fuse Quickbird images and IKONOS images.

Generally, Image fusion is performed at three different levels at which the fusion takes place: pixel, feature, and decision level [5]. Nowadays, the technique of Image fusion is used in many areas such as remote sensing and medical imaging which leads to enhance interpretation by a human observer. The paper is organized into six sections where Section 2 describes the Image Fusion based on Top-hat Transform; Section 3 describes the Image Fusion based on Wavelet Transformation; Section 4 deals with Quality Assessment Techniques; Section 5 deals with Results and Discussions; and Section 6 deals with Conclusions.

\section{Image fusion based on top-hat transform}

Morphology is a broad set of image processing operations that process images based on shapes. Morphological operations apply a structuring element to an input image, creating an output image of the same size. In a morphological operation, the value of each pixel in the output image is based on a comparison of the corresponding pixel in the input image with its 
neighbors. By choosing the size and shape of the neighborhood, a morphological operation that is sensitive to specific shapes in the input image can be constructed. The most basic morphological operations are dilation and erosion. Dilation adds pixels to the boundaries of objects in an image, while Erosion removes pixels on object boundaries. The number of pixels added or removed from the objects in an image depends on the size and shape of the structuring element used to process the image. In the morphological dilation and erosion operations, the state of any given pixel in the output image is determined by applying a rule to the corresponding pixel and its neighbors in the input image. The rule used to process the pixels defines the operation as dilation or erosion.

An essential part of the dilation and erosion operations is the structuring element that is used to probe the input image. A structuring element is a matrix consisting of only 0 's and 1's that can have any arbitrary shape and size. The pixels with values of 1 define the neighborhood. Twodimensional or flat, structuring elements are typically much smaller than the image being processed. The center pixel of the structuring element, called the origin, identifies the pixel of interest - the pixel being processed. The pixels in the structuring element containing 1's define the neighborhood of the structuring element. These pixels are also considered in dilation or erosion processing.

In Image Processing, top-hat transform is an operation that extracts small elements and details from the input images. Top-hat transforms are used for various image processing tasks, such as feature extraction, background equalization, image enhancement, image fusion, etc. There are two types of top-hat transform: white top-hat transform and black top-hat transform. The white top-hat transform is defined as the difference between the input image and its opening by some structuring element. The white top-hat transform returns an image, containing those "objects" or "elements" of an input image that are "smaller" than the structuring element and are brighter than their surroundings. The black top-hat transform is defined as the difference between the closing and the input image. The black top hat returns an image, containing those "objects" or "elements" of an input image that are "smaller" than the structuring element, and are darker than their surroundings.

In Matlab, the function 'imtophat' performs morphological top-hat filtering on the grayscale or binary input image. Top-hat filtering computes the morphological opening of the image and then subtracts the result from the original image. This function uses the structuring element. It must be a single structuring element object, not an array containing multiple structuring element objects.

\section{Image fusion based on wavelet transformation}

Image fusion using Wavelet transformations provides multiscale and multiresolution analysis functions. Wavelet transform is a linear tool in its original form [6]. The Wavelet technique outperforms the standard fusion technique in spatial and spectral quality. The Decimated wavelet transformations are Discrete Wavelet Transformations (DWT)[7] and the Undecimated Wavelet Transformations are Stationary Wavelet Transformations (SWT). There are three general classes of Wavelets - Orthogonal, Biorthogonal, and Nonorthogonal in both DWT and SWT. The Wavelet filters used for Orthogonal wavelets are Haar and Daubechies, for Biorthogonal wavelets are Bior1.1, Bior1.3, Bior1.7, and Nonorthogonal wavelets are Meyers, Coiflets, and Symlets. The 2-D discrete wavelet transformation is used for fusing images. In this paper, the five wavelet fusion methods are discussed i.e., top-hat transformation, orthogonal wavelet fusion with decimation (ORTH), biorthogonal wavelet 
fusion with decimation (BIOR), orthogonal wavelet fusion with undecimation (UORTH), and biorthogonal wavelet fusion with undecimation (UBIOR).

Perform histogram match process between PAN and MS images to obtain three new PAN images. Use the Wavelet Transform to decompose new PAN images and different bands of MS images. Add the detailed images of the decomposed PAN image at different levels to the corresponding details of different bands in the MS image and obtain the new details component in the different bands of the MS image. Perform Inverse Wavelet Transform on the bands of MS images respectively and obtain the fused image [8].

\section{Quality assessment techniques}

To assess the quality of fused images, some quality performance measures are required. While visual inspection has limitations due to human judgment, the quantitative approach based on the evaluation of "distortion" in the resulting fused image is more desirable for mathematical modeling [9].

In Mathematical modeling, the quantitative measure is desirable. Quantitative measures are used to predict the perceived image quality. In this study, quality assessment using noisebased measures is used to evaluate the noise of the fused image by comparing it to its original MS image. The following optimal noise-based measures are implemented to judge the performance of the above-discussed fusion methods [10][11].

a) Entropy: This is used to quantify the quantity of information contained in the fused image. A bigger value shows good fusion results.

$$
H=-\sum_{i=0}^{L-1} h_{F}(i) \log _{2} h_{F}(i)
$$

where $\mathrm{h}_{\mathrm{F}}$ is the normalized histogram of the fused image and $\mathrm{L}$ is the no. of gray levels.

b) Correlation Coefficient $(\boldsymbol{C C})$ : This is used to measure the similarities of the fused image to the corresponding original images.

$$
\mathrm{CC}=\frac{\mathrm{E}(\mathrm{XY})-\mathrm{E}(\mathrm{X}) \mathrm{E}(\mathrm{Y})}{\sqrt{\mathrm{E}\left(\mathrm{X}^{2}\right)-\mathrm{E}^{2}(\mathrm{X})} \sqrt{\mathrm{E}\left(\mathrm{Y}^{2}\right)-\mathrm{E}^{2}(\mathrm{Y})}}
$$

c) Mean squared error (MSE): This is used to measure the spectral distortion.

$$
M S E=\frac{\sum_{i=1}^{M} \sum_{j=1}^{N}\left(I_{R}(i, j)-I_{F}(i, j)^{2}\right)}{M * N}
$$

where $I_{R}(i, j)$ denotes pixel $(i, j)$ of the image reference and $I_{F}(i, j)$ denotes pixel $(i, j)$ of the fused image, $\mathrm{M}^{*} \mathrm{~N}$ is the image size.

d) Peak signal to signal noise ratio (PSNR): This is used to reveal the radiometric distortion of the final image compared to the original image.

$$
\operatorname{PSNR}(d B)=10 \log _{10}\left(\frac{P e a k}{M S E 2}\right)
$$

where the peak is the maximum possible pixel value. Peak equals 255 for 8-bit images 2047 for 11-bit images and 65535 for 16bit images.

e)Root mean squared error (RMSE): This is used to measure the standard error of the fused image.

$$
R M S E=\sqrt{M S E}
$$


f) Mean absolute error (MAE): This is used to measure the average magnitude of the errors in a set of forecasts, without considering their direction. It measures accuracy for continuous variables.

$$
M A E=\frac{1}{M * N} \sum_{x=0}^{M-1} \sum_{y=0}^{N-1}\left(I_{R}(x, y)-I_{F}(x, y)\right)
$$

where $I_{R}(x, y)$ denotes pixel $(x, y)$ of the image reference and $I_{F}(x, y)$ denotes pixel $(x, y)$ of the fused image, $\mathrm{M}^{*} \mathrm{~N}$ is the image size.

g) Mutual Information Measure(MIM): This is used to furnish the amount of information of one image in another. Given two images $\mathrm{M}(\mathrm{i}, \mathrm{j})$ and $\mathrm{N}(\mathrm{i}, \mathrm{j})$, MIM is defined as:

$$
I_{M N}=\sum_{x, y} P_{M N}(x, y) \log \frac{P_{M N}(x, y)}{P_{M}(x) P_{N}(y)}
$$

where $\mathrm{P}_{\mathrm{M}}(\mathrm{x})$ and $\mathrm{P}_{\mathrm{N}}(\mathrm{y})$ are the probability density functions in the individual images and $\mathrm{P}_{\mathrm{MN}}(\mathrm{x}, \mathrm{y})$ is the joint probability density function.

h) Fusion Factor $(\boldsymbol{F F})$ : This is defined as $\mathrm{FF}=\mathrm{I}_{\mathrm{AF}}+\mathrm{I}_{\mathrm{BF}}$ where $\mathrm{A}$ and $\mathrm{B}$ are given two images and $\mathrm{F}$ is the fused image. A higher value of FF indicates that the fused image contains a moderately good amount of information present in both images.

i) Fusion symmetry(FS): This is used to indicate the degree of symmetry in the information content from both images.

$$
F S=a b s\left(\frac{I_{A F}}{I_{A F}+I_{B F}}-0.5\right)
$$

The quality of the fusion technique depends on FS. When sensors are of good quality, FS should be as low as possible so that the fused image derives features from both input images. If any sensors are of low quality then it is better to maximize.

j) Fusion Index(FI): This is defined as $\mathrm{FI}=\mathrm{I}_{\mathrm{AF}} / \mathrm{I}_{\mathrm{BF}}$ where $\mathrm{I}_{\mathrm{AF}}$ is the mutual information index between MS image and fused image and $\mathrm{I}_{\mathrm{BF}}$ is the mutual information index between PAN image and fused image. The quality of the fusion technique depends on the degree of the Fusion index.

\section{Results and discussions}

The above discussed six fusion methods were implemented and executed on PC with $2.4 \mathrm{G}$ CPU and 2.0 G RAM using Matlab 7.6.0 to compare their fusion results. The experiment was conducted and tested on IRS-1D PAN and MS images for the locations Vishakhapatnam and Hyderabad, on QuickBird image data and Los Angeles image data, and the following results were evaluated.

The following are the fused images for the location Vishakhapatnam using the methods top-hat transform, ORTH, BIOR, UORTH, and UBIOR. 


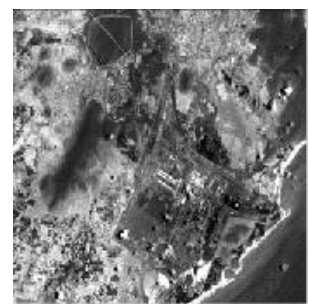

Figure 1. PAN

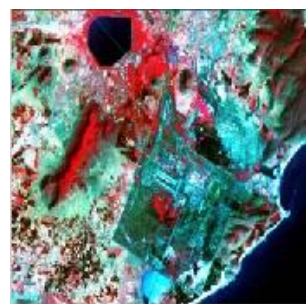

Figure 2. MS

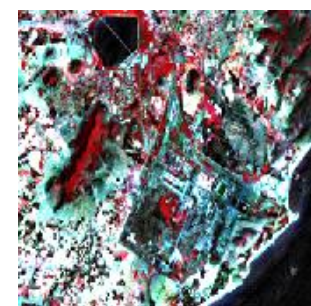

Figure 3. Top-hat

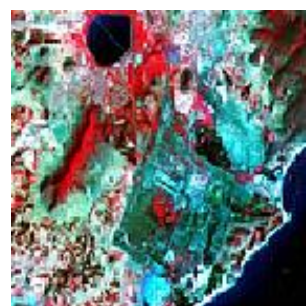

Figure 4. ORTH

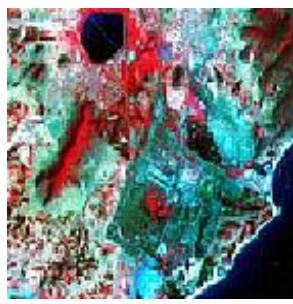

Figure 5. BIOR

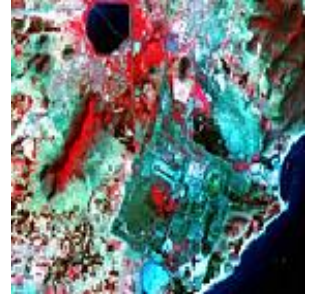

Figure 6. UORTH

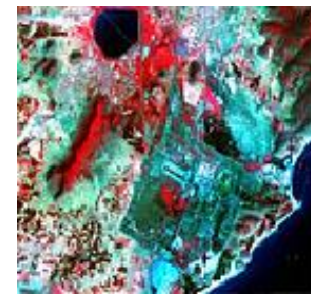

Figure 7. UBIOR

Table 1. Comparison of different metrics using different methods for VISHAKHAPATNAM image

\begin{tabular}{|c|c|c|c|c|c|}
\hline Metrics & TOP-HAT & ORTH & BIOR & UORTH & UBIOR \\
\hline SD & 70.391 & 32.680 & 32.410 & 32.086 & 31.696 \\
\hline Entropy & 7.6795 & 7.7677 & 7.7728 & 7.7840 & 7.8048 \\
\hline CC & 0.8108 & 0.9671 & 0.9651 & 0.9806 & 0.9811 \\
\hline MSE & 0.0116 & 0.0117 & 0.0120 & 0.0094 & 0.0092 \\
\hline PSNR & 67.4786 & 67.459 & 67.353 & 68.400 & 68.475 \\
\hline RMSE & 0.1078 & 0.1080 & 0.1094 & 0.0969 & 0.0961 \\
\hline MAE & 0.0459 & 0.0820 & 0.0830 & 0.0741 & 0.0735 \\
\hline MIM & 0.7015 & 1.0995 & 1.0690 & 1.2081 & 1.1931 \\
\hline FF & 1.4031 & 2.1991 & 2.1379 & 2.4162 & 2.3862 \\
\hline FS & 0.0334 & 0.1387 & 0.1405 & 0.1481 & 0.1524 \\
\hline FI & 0.8747 & 0.5656 & 0.5612 & 0.5430 & 0.5329 \\
\hline
\end{tabular}

The following are the fused images about the location Hyderabad using the methods tophat transform, ORTH, BIOR, UORTH, and UBIOR. 


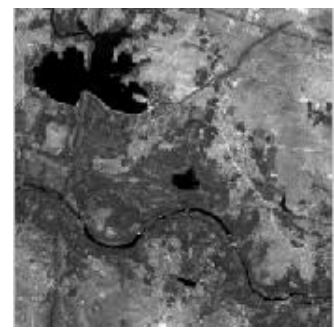

Figure 8. PAN

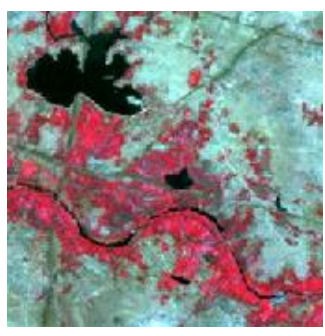

Figure 9. MS

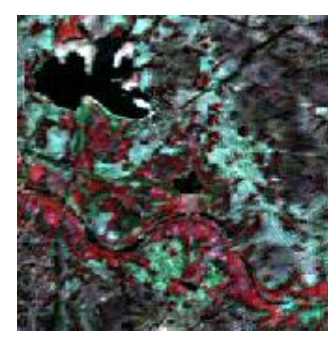

Figure 10. Top-hat

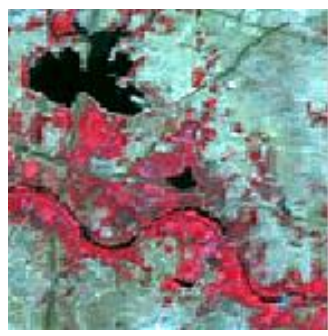

Figure 11. ORTH

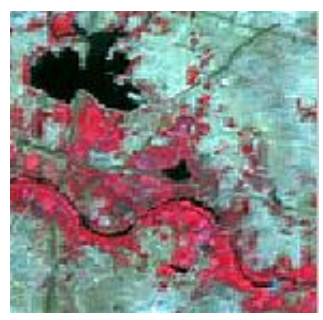

Figure 12. BIOR

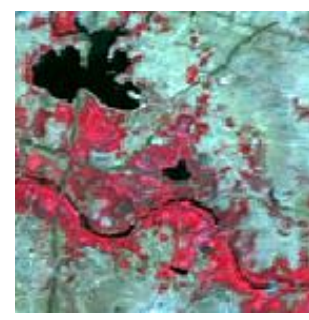

Figure 13. UORTH

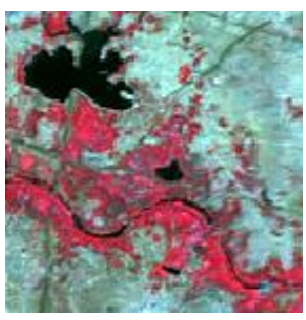

Figure 14. UBIOR

Table 2. Test case 2 for comparison of different metrics using different methods for HYDERABAD image

\begin{tabular}{|c|c|c|c|c|c|}
\hline Metrics & TOP-HAT & ORTH & BIOR & UORTH & UBIOR \\
\hline SD & 43.279 & 21.001 & 20.854 & 20.709 & 20.471 \\
\hline Entropy & 7.3523 & 7.1814 & 7.2059 & 7.1395 & 7.1559 \\
\hline CC & 0.6431 & 0.9640 & 0.9619 & 0.9778 & 0.9787 \\
\hline MSE & 0.0242 & 0.0061 & 0.0062 & 0.0052 & 0.0051 \\
\hline PSNR & 64.3243 & 70.248 & 70.173 & 70.988 & 71.059 \\
\hline RMSE & 0.1550 & 0.0784 & 0.0790 & 0.0720 & 0.0714 \\
\hline MAE & 0.0757 & 0.0617 & 0.0622 & 0.0569 & 0.0564 \\
\hline MIM & 0.3212 & 1.1660 & 1.1420 & 1.2743 & 1.2676 \\
\hline FF & 0.6423 & 2.3321 & 2.2839 & 2.5487 & 2.5353 \\
\hline FS & 0.0172 & 0.0952 & 0.1000 & 0.1107 & 0.1179 \\
\hline FI & 0.9335 & 0.6800 & 0.6666 & 0.6375 & 0.6183 \\
\hline
\end{tabular}

The following are the fused images of QuickBird image data using the methods top-hat transform, ORTH, BIOR, UORTH, and UBIOR. 


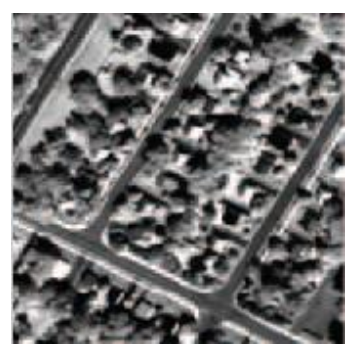

Figure 15. PAN

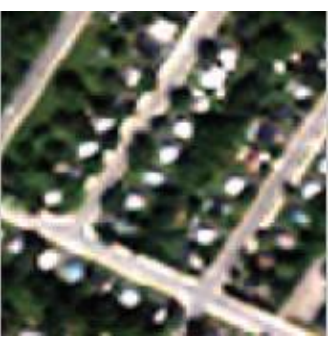

Figure 16. MS

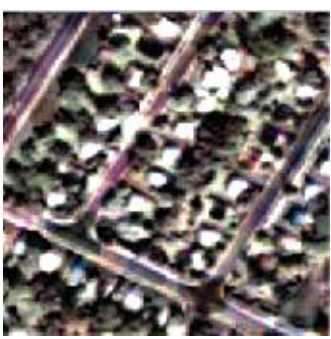

Figure 17. Top-hat

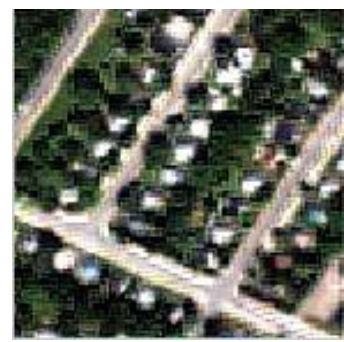

Figure 18. ORTH

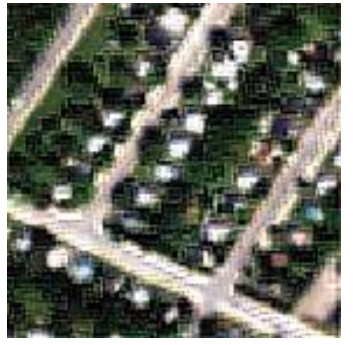

Figure 19. BIOR

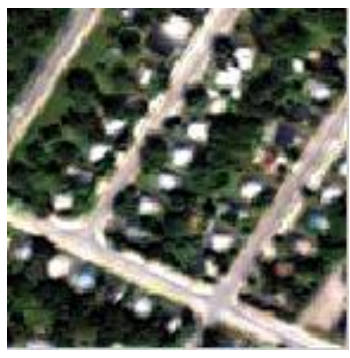

Figure 20. UORTH

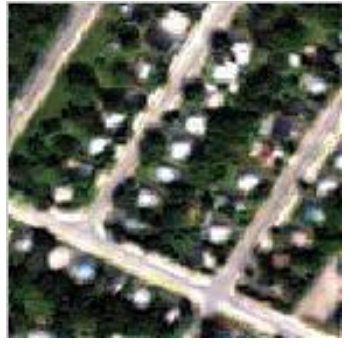

Figure 21. UBIOR

Table 3. Test case 3 for comparison of different metrics using different methods for QuickBird image

\begin{tabular}{|c|c|c|c|c|c|}
\hline Metrics & TOP-HAT & ORTH & BIOR & UORTH & UBIOR \\
\hline SD & 73.4379 & 41.458 & 41.251 & 40.305 & 20.471 \\
\hline Entropy & 7.6893 & 7.5449 & 7.5643 & 7.6332 & 7.1559 \\
\hline CC & 0.5269 & 0.9480 & 0.9448 & 0.9755 & 0.9787 \\
\hline MSE & 0.0299 & 0.0143 & 0.0141 & 0.0095 & 0.0051 \\
\hline PSNR & 63.3749 & 66.580 & 66.653 & 68.352 & 71.059 \\
\hline RMSE & 0.1729 & 0.1195 & 0.1185 & 0.0975 & 0.0714 \\
\hline MAE & 0.0761 & 0.0935 & 0.0926 & 0.0770 & 0.0564 \\
\hline MIM & 0.3546 & 0.6786 & 0.6436 & 0.8690 & 1.2676 \\
\hline FF & 0.7093 & 1.3572 & 1.2871 & 1.7380 & 2.5353 \\
\hline FS & 0.1306 & 0.3584 & 0.3865 & 0.3575 & 0.1179 \\
\hline FI & 1.7071 & 0.1650 & 0.1280 & 0.1661 & 0.6183 \\
\hline
\end{tabular}

The following are the fused images of Losangels image data using the methods top-hat transform, ORTH, BIOR, UORTH, and UBIOR. 


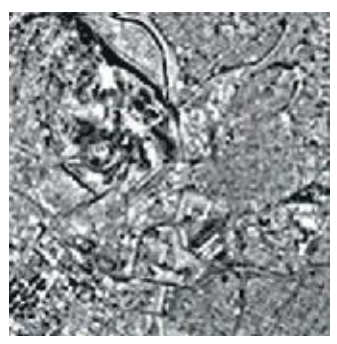

Figure 22. PAN

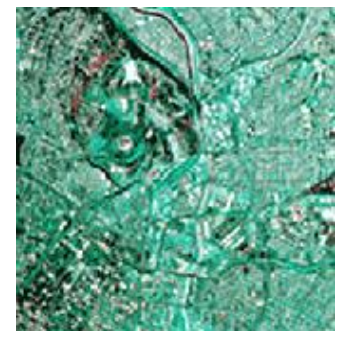

Figure 23. MS

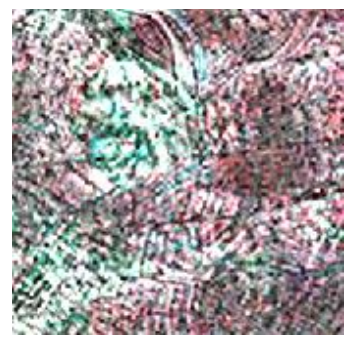

Figure 24. Top-hat

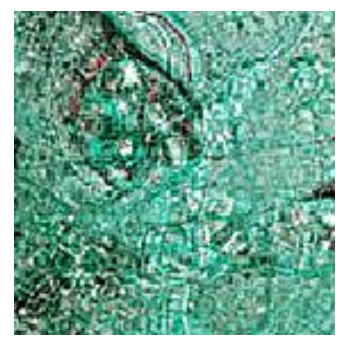

Figure 25. ORTH

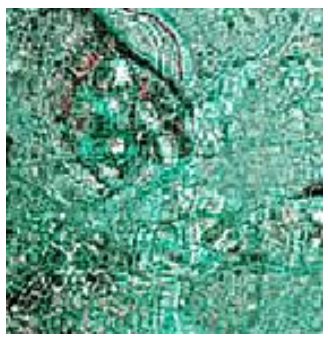

Figure 26. BIOR

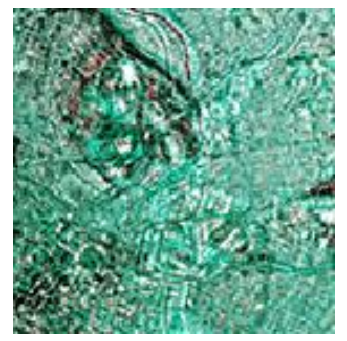

Figure 27. UORTH

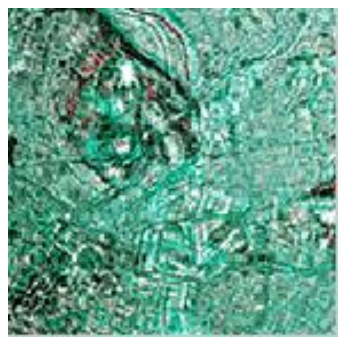

Figure 28. UBIOR

Table 4. Test case 3 for comparison of different metrics using different methods for LosAngels image

\begin{tabular}{|c|c|c|c|c|c|}
\hline Metrics & TOP-HAT & ORTH & BIOR & UORTH & UBIOR \\
\hline SD & 63.3069 & 28.595 & 28.750 & 26.957 & 26.800 \\
\hline Entropy & 7.4040 & 7.6149 & 7.6121 & 7.6290 & 7.6292 \\
\hline CC & 0.5847 & 0.8659 & 0.8621 & 0.9113 & 0.9172 \\
\hline MSE & 0.0357 & 0.0211 & 0.0216 & 0.0161 & 0.0155 \\
\hline PSNR & 62.6066 & 64.885 & 64.785 & 66.068 & 66.222 \\
\hline RMSE & 0.1889 & 0.1453 & 0.1470 & 0.1268 & 0.1246 \\
\hline MAE & 0.0723 & 0.1166 & 0.1175 & 0.1038 & 0.1021 \\
\hline MIM & 0.1861 & 0.3401 & 0.3322 & 0.4180 & 0.4303 \\
\hline FF & 0.3721 & 0.6802 & 0.6643 & 0.8360 & 0.8606 \\
\hline FS & 0.2578 & 0.4191 & 0.4423 & 0.4236 & 0.4479 \\
\hline FI & 3.1283 & 0.0881 & 0.0612 & 0.0827 & 0.0549 \\
\hline
\end{tabular}

\section{Conclusions}

The various fusion results are analyzed by using quality performance metrics. S.D is minimum for Vishakhapatnam, Hyderabad, Quick Bird, and Losangels images using UBIOR. The higher value for Entropy is achieved for Vishakhapatnam, Hyderabad, and Losangels images using UBIOR. CC is maximum for Vishakhapatnam, Hyderabad, Quick Bird, and Losangels images using UBIOR. The higher the value of the correlation coefficients, the more similar is the fused image to the corresponding original MS image. PSNR and FS are greater for Vishakhapatnam, Hyderabad, Quick Bird, Losangels images using UBIOR. The higher value of PSNR implies that the spectral information in the MS image was preserved 
effectively. MSE, RMSE, FI is smaller for Vishakhapatnam, Hyderabad, Quick Bird, and Losangels images using UBIOR. MAE is minimum for Vishakhapatnam and Losangels images using Top-hat transform. MAE is minimum for Hyderabad and Quick Bird images using UBIOR. MIM, FF is more for Vishakhapatnam, Hyderabad, and Quick Bird images using UORTH. MIM, FF is more for Losangels images using UBIOR. MIM and FS are more for Losangels images using UBIOR. The higher values of MM, FS, and FF indicate that symmetry is achieved by retaining spectral information. Hence, it is ascertained that the Undecimated Biorthogonal Wavelet Transform method has superior performance than Tophat transforms, decimated orthogonal wavelet transform, decimated biorthogonal wavelet transform, and undecimated orthogonal wavelet transform. The results are verified for a few satellite images only and the study can be extended for other types of images.

\section{References}

[1] P. J. Burt and R. J. Lolezynski, "Enhanced image capture through fusion", in Proceedings of the Fourth International Conference on Computer Vision, Berlin, Germany, pp.173-182

[2] H. Li, B. Manjunath and S. Mitra, "Multisensor image fusion using the wavelet transform", Graph, Models Image Process, vol.57, no.3, pp.235-245

[3] X. Yang, W. Yang, and J. Pei, "Different focus points images fusion based on wavelet decomposition", Proceedings of Third International Conference on Information Fusion, vol.1, pp.3-8, (2000)

[4] Z. Zhang and R. S. Blum, "Image fusion for a digital camera application", Conference Record of the ThirtySecond Asilomar Conference on Signals, Systems and Computers, vol.1, pp.603-607, (1998)

[5] Lehigh Univ: http://www.eecs.lehigh.edu/SPCRL/IF/image_fusion.htm

[6] S. Mallat, "A wavelet tour of signal processing", Academic Press, London, (1998)

[7] Z. Li, Z. Jing, X. Yang, and S. Sun, "Color transfer based Remote Sensing Image Fusion using nonseparable Wavelet Frame Transform”, Pattern Recognition Letters, vol.26, no.13, pp.2006-2014, (2005)

[8] G. Hong and Y. Zhang, "Comparison and Improvement of wavelet-based Image fusion", International Journal of Remote Sensing, Vol. 29, No. 3, pp. 673-69, Feb.(2008).

[9] W. Shi., C. Zhu., Tian, and J. Nichol, "Wavelet-based image fusion and quality assessment", International Journal of Applied Earth Observation and Geoinformation, vol.6, pp.241-251, (2005)

[10] S. Li, Z. Li, J. Gong, "Multivariate statistical of measures for assessing the quality of image fusion", International Journal of Image and Data Fusion, (2010)

[11] Yuhendra, "Wavelet PCA based images fusion techniques and quality assessment" 